\title{
The UK Diabetic Retinopathy Electronic Medical Record (UK DR EMR) Users Group, Report 2: real- world data for the impact of cataract surgery on diabetic macular oedema
}

\author{
Alastair K Denniston, ${ }^{1,2}$ Usha Chakravarthy, ${ }^{3}$ Haogang Zhu, ${ }_{1}^{4}$ Aaron Y Lee, ${ }^{5}$ \\ David P Crabb, ${ }^{4}$ Adnan Tufail, ${ }^{2}$ Clare Bailey, ${ }_{1}^{6}$ Toks Akerele, ${ }^{7}$ Sahar Al-Husainy, ${ }^{8}$ \\ Christopher Brand, ${ }^{9}$ Louise Downey, ${ }_{1}^{10}$ Alan Fitt, ${ }^{11}$ Rehna Khan, ${ }^{12}$ Vineeth Kumar, ${ }^{13}$ \\ Aires Lobo, ${ }^{14}$ Sajjad Mahmood, ${ }_{1}^{15}$ Kaveri Mandal, ${ }^{16}$ Martin Mckibbin, ${ }^{17}$ \\ Geeta Menon, ${ }^{18}$ Salim Natha, ${ }_{1}^{19}$ Jong Min Ong, ${ }^{20}$ Marie D Tsaloumas, ${ }^{1}$ Atul Varma, ${ }^{21}$ \\ Elizabeth Wilkinson, ${ }^{22}$ Robert L Johnston, ${ }^{23}$ Catherine A Egan, ${ }^{2}$ on behalf of the UK DR \\ EMR Users Group
}

- Additional material is published online only. To view please visit the journal online (http://dx.doi.org/10.1136/ bjophthalmol-2016-309838).

For numbered affiliations see end of article.

\section{Correspondence to} Catherine A Egan, Moorfields Eye Hospital NHS Foundation Trust, 162 City Road, London, EC1V 2PD, UK: Catherine. Egan@moorfields.nhs.uk

Received 31 October 2016 Revised 14 March 2017

Accepted 19 March 2017 Published Online First 9 May 2017

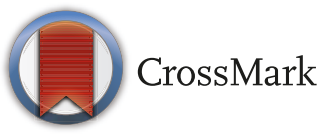

To cite: Denniston AK, Chakravarthy $\mathrm{U}$, Zhu $\mathrm{H}$, et al. Br J Ophthalmol

2017:101:1673-1678.

\section{ABSTRACT}

Aim To assess the rate of 'treatment-requiring diabetic macular oedema (DMO)' in eyes for the two years before and after cataract surgery.

Methods Multicentre national diabetic retinopathy (DR) database study with anonymised data extraction across 19 centres from an electronic medical record system. Inclusion criteria: eyes undergoing cataract surgery in patients with diabetes with no history of DMO prior to study start. The minimum dataset included: age, visual acuity (all time-points), injection episodes, timing of cataract surgery and ETDRS grading of retinopathy and maculopathy. Main outcome measure: rate of developing first episode of treatment-requiring $\mathrm{DMO}$ in relation to timing of cataract surgery in the same eye.

Results 4850 eyes met the inclusion criteria. The rate of developing treatment-requiring DMO in this cohort was $2.9 \%$ in the year prior to surgery versus $5.3 \%$ in the year after surgery $(p<0.01)$. The risk of 'treatment-requiring $\mathrm{DMO}^{\prime}$ increased sharply after surgery, peaking in the 3-6 months' period (annualised rates of $5.2 \%, 6.8 \%, 5.6 \%$ and $4.0 \%$ for the $0-3,3-6,6-9$ and $9-12$ months' post-operative time periods respectively). Risk was associated with pre-operative grade of retinopathy: risk of DMO in the first year post-operatively being 1.0\% (no DR pre-operatively), 5.4\% (mild non-proliferative diabetic retinopathy; NPDR), 10.0\% (moderate NPDR), 13.1\% (severe NPDR) and $4.9 \%(P D R)(p<0.01)$.

Conclusions This large real-world study demonstrates that the rate of developing treatment-requiring DMO increases sharply in the year after cataract surgery for all grades of retinopathy, peaking in the 3-6 months' postoperative period. Patients with moderate and severe NPDR are at particularly high risk.

\section{INTRODUCTION}

Visually significant cataract is a common problem in people with diabetes, occurring earlier and with a prevalence of 2-5 times that of the population without diabetes. ${ }^{1-3}$ Cataract surgery is known to lead to increased levels of inflammatory mediators such as prostaglandins, thromboxane $\mathrm{A}$, nitric acid and various cytokines in addition to vascular endothelial growth factor (VEGF) ${ }^{4-7}$ Patel et al l $^{4}$ showed that some of these changes are present within 1 day of surgery and do not return to normal for several weeks. Retinovascular changes also occur, including vascular hyperpermeability and leukostasis. ${ }^{4-68}$

Despite these recognised changes in the ocular microenvironment, clinical studies are inconclusive as to the effect of cataract surgery on the onset of diabetic macular oedema (DMO). Furthermore there is quite marked variation in the rates of DMO reported in such studies, which may reflect study design, variable definition of $\mathrm{DMO}$, and the population sampled. Most studies in this area are small with fewer than 100 patients. ${ }^{9-13}$ An important exception is ETDRS Report 25 which analysed the subset of 270 eyes (205 patients) who underwent cataract surgery during the course of the prospective ETDRS randomised controlled trial (RCT). These patients with non-proliferative diabetic retinopathy (NPDR) had mild to severe or early proliferative diabetic retinopathy (PDR) and/or macular oedema. This was a post-hoc analysis of patients who happened to undergo cataract surgery during the study period without standardisation of timing or perioperative management. With regard to macular oedema the defined endpoint was clinically significant macular oedema (CSME), which was defined without the use of optical coherence tomography (OCT) imaging and was based on the clinical endpoint of stereoscopic fundus photography. This study showed no significant increase in the rate of CSME with 29\% of patients having CSME at the study visit prior to cataract surgery and 31\% having CSME post-surgery. ${ }^{9} \mathrm{~A}$ more recent prospective study looking at unilateral cataract surgery in 132 eyes (with the fellow eye acting as control) noted a trend towards a higher rate of CSME in the operated eye versus the fellow eye $(6.1 \%$ vs $4.5 \%)$. In this study all patients had 3 monthly fluorescein angiography; 
OCT was not performed in all patients but only those with suspected CSME. ${ }^{12}$

The use of electronic medical record (EMR) systems which routinely gather clinically relevant data provides the opportunity to analyse larger study cohorts than would be practical within a clinical trial. In the UK, the most widely-used ophthalmic EMR has implemented a nationally-defined minimum dataset for diabetic retinopathy (DR) that mandates recording of the minimum clinical signs necessary to allow an algorithm within the software to automatically calculate a precise proxy-ETDRS/ International Clinical Grading System retinopathy and maculopathy grade after each consultation (as described in UK DR EMR Report 1 appendix 1). ${ }^{14}$ All interventions including laser procedures, intravitreal injections and ophthalmic operations are recorded, providing the opportunity to assess whether the rate of treatment-requiring DMO is influenced by cataract surgery in a large 'real-world' cohort. In the UK, the National Health Service (NHS) use of ranibizumab is regulated by the National Institute for Health and Care Excellence (NICE), with treatment only being funded for DMO where it is demonstrated on OCT to be associated with a central macular thickness (CMT) of over 400 microns. ${ }^{15}$ It is important to note that CMT is not precisely defined as, for example, subfoveal thickness or central grid thickness. Random audits of ranibizumab use by commissioners have the potential to withdraw funding, so there is a strong motivation for physicians to comply with the guidance. It is also important to note that there is no visual acuity level specified in the NICE guidance. Due to this unique regulatory framework, the assessment of 'Ranibizumab-requiring DMO' in the UK therefore provides a surrogate for an OCT-defined threshold of DMO severity.

\section{METHODS}

\section{Ethical approval}

The lead clinician and Caldicott Guardian at each centre gave written approval for extraction of anonymised data. The study protocol was approved by the head of research governance at the lead clinical centre. This study was conducted in accordance with the Declaration of Helsinki and the UK Data Protection Act.

\section{Data collection}

Anonymised data were remotely extracted from 19 centres using the same EMR system (Medisoft Ophthalmology, Medisoft, Leeds, UK). Each site is the only NHS provider of DMO care to their local population and very few patients switch between providers or access care privately. Data were extracted through the EMR compulsory DR structured assessment module. The minimum dataset included: age, visual acuity at baseline and at all subsequent visits, injection episodes, timing of cataract surgery and proxy-ETDRS/International Clinical Grading system grading of retinopathy and maculopathy.

\section{Inclusion criteria}

Eyes undergoing cataract surgery from patients with diabetes, with retinopathy grade(s) recorded in the 2 years pre-surgery and post-surgery, and who had not developed DMO requiring treatment prior to study entry (ie, 2 years pre-surgery). For the purposes of this study we analysed the development of DMO in terms of the requirement for treatment, that is DMO of sufficient severity to warrant an intervention; this is referred to as 'treatment-requiring DMO' throughout the study. It is recognised that some of these cases might have spontaneously resolved without treatment, but the term 'treatment-requiring' is used to indicate that the severity of DMO was considered sufficient by the attending clinician to institute treatment. The EMR mandated structured assessment only requires recording of the location of retinal thickening, not the causative factor(s). Recorded post-operative episodes of retinal thickening in patients with diabetes may therefore include some cases of pseudophakic macular oedema (post-operative, cystoid macular oedema). In line with the study aims of providing a 'real world' analysis, patients were included even if they had additional pre-operative risk factors (such as history of uveitis) or intra-operative complications.

\section{Analysis}

The primary outcome measure was the rate of developing first episode of treatment-requiring DMO in relation to timing of cataract surgery in the same eye, described both as incident risk and as cumulative risk over each successive year. Subsidiary analyses included effect of level of retinopathy on the rate of developing treatment-requiring DMO in the same eye in relation to cataract surgery. Survey of practice included analysis of the provision of type of treatments used for DMO and their timing.

\section{RESULTS}

\section{Baseline characteristics}

Participants

Data were extracted on 123968 eyes of 61984 patients with diabetic retinopathy grades (including a grade of no diabetic retinopathy) in the EMR. There were 33967 male patients, 28002 female patients and 15 cases of unrecorded gender.

The inclusion criteria were met for 4850 eyes from 3837 patients, namely eyes undergoing cataract surgery in patients with diabetes, with retinopathy grade(s) recorded in the 2 years pre-sugery and post-surgery, and who had not developed DMO requiring treatment prior to study entry (ie, 2 years pre-surgery). Treatments for DMO included macular laser, injection of intravitreal anti-VEGF therapies (ranibizumab, bevacizumab, aflibercept) and injection of intravitreal corticosteroids (triamcinolone, Iluvien and Ozurdex).

The study group comprised 1719 eyes with no apparent DR, 1034 with mild NPDR, 1527 with moderate NPDR, 165 with severe NPDR, and 405 with proliferative DR.

\section{Rate of developing first episode of treatment-requiring DMO in relation to cataract surgery}

The rate of developing first episode of treatment-requiring DMO in the whole cohort was $3.1 \%$ and $2.9 \%$ in each of the 2 years prior to surgery versus $5.3 \%$ and $4.8 \%$ in each of the 2 years after surgery $(p<0.01$; see table 1$)$. More detailed analysis by 3-month intervals demonstrated that the risk of 'treatment-requiring DMO' increased sharply after surgery, peaking in the 3-6 months post-operative period (figure 1). The cumulative risk rose from $6.2 \%$ for the 24 -month pre-operative period to $14.7 \%$ for the full 48 -month peri-operative period (see table 2).

\section{Effect of retinopathy grade on the risk of developing first episode of 'Treatment-requiring DMO' in relation to cataract surgery}

The risk of developing 'Treatment-requiring DMO' was significantly associated with pre-operative grade of retinopathy. In the first year after cataract surgery the risk of developing first episode of 'Treatment-requiring DMO' was 1.0\% (no DR seen pre-operatively), 5.4\% (mild NPDR), 10.0\% (moderate NPDR), 13.1\% (severe NPDR) and 4.9\% (PDR) (table 1, figure 2). For 
Table 1 Risk of Developing First Episode of Treatment-Requiring diabetic macular oedema (DMO)

\begin{tabular}{|c|c|c|c|c|c|}
\hline & Time in relation to cataract surgery (months) & -24 to -12 & -12 to 0 & 0 to +12 & +12 to +24 \\
\hline \multirow[t]{7}{*}{ Risk of DMO (\%) } & All eyes $(n=4850)$ & 3.1 & 2.9 & 5.3 & 4.8 \\
\hline & Stratified by ETDRS grade* & & & & \\
\hline & No $D R(n=1719)$ & 0.0 & 0.0 & 1.0 & 0.8 \\
\hline & Mild NPDR $(n=1034)$ & 2.1 & 2.6 & 5.4 & 5.6 \\
\hline & Moderate NPDR $(n=1527)$ & 5.4 & 5.5 & 10.0 & 8.9 \\
\hline & Severe NPDR $(n=165)$ & 11.5 & 11.0 & 13.1 & 11.5 \\
\hline & PDR $(n=405)$ & 5.9 & 4.2 & 4.9 & 4.2 \\
\hline
\end{tabular}

*Stratification is by proxy-ETDRS/International Clinical Grading system grade of retinopathy at most recent assessment prior to cataract surgery (mild, moderate and severe NPDR and PDR retinopathy). Time periods are up to but not including the upper boundary, ie, -24 to -12 equates to any time point from -24 months to 1 day prior to -12 months. An event occurring at exactly -12 months is included in the -12 to 0 month group. Similarly, patients treated on the day of surgery are included in the $0-12$ month group. Since development of DMO is a censoring event, the number of eyes at risk reduces over time as follows: 4850 ( -24 to -12 months), 4702 ( -12 to 0 months), 4565 (0 to +12 months), 4323 (+12 to +24 months).

NPDR, non-proliferative diabetic retinopathy; PDR, proliferative diabetic retinopathy.

mild and moderate grades of retinopathy this represented an approximate doubling of the risk of DMO compared with the year prior to surgery; the relative increase in risk was less great for severe NPDR and for PDR, but it is noteworthy that the risk of DMO in the severe NPDR group was more than twice that of the PDR group.

\section{Cumulative risk of developing Treatment-Requiring DMO over time}

The cumulative risk of developing 'Treatment-requiring DMO' was calculated according to the level of retinopathy prior to surgery. The cumulative risk varied according to retinopathy grade and time in relation to surgery. It should be noted that as per the study inclusion criteria no patients had 'treatment-requiring DMO' at their baseline (defined as 2 years prior to cataract surgery). Cumulative risk of developing first episode of 'Treatment-requiring DMO' at 4 years of follow-up (ie, 2 years post cataract surgery) was $14.7 \%$ for the whole cohort, but varied significantly according to retinopathy being $1.4 \%$ (no DR seen pre-operatively), 13.4\% (mild NPDR), 29.5\% (moderate NPDR), 59\% (severe NPDR) and 19.1\% (PDR) (table 2, figure 2).

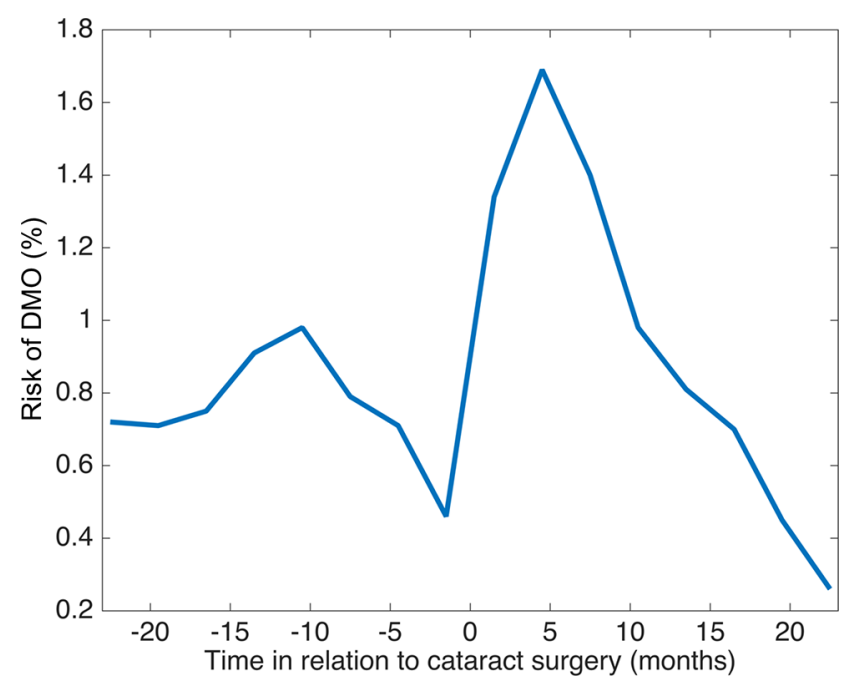

Figure 1 Risk of developing first episode of 'Treatment-requiring diabetic macular oedema (DMO)' in relation to cataract surgery. Risk of developing first episode of 'Treatment-requiring DMO' in patients with diabetes in the 2 years before and after cataract surgery. Percentages given are of risk of 'treatment-requiring DMO' in that 3-month period.

\section{Treatment modalities for DMO}

Of the 4850 eyes included in this study, 622 received treatment for the first episode of 'Treatment-requiring DMO' during the study period (ie, up to 2 years post cataract surgery). Macular laser was the predominant therapy used both before and after cataract surgery, comprising $84.6 \%$ vs $68.5 \%$ of all first treatments for DMO in the 2 years pre-surgery versus 2 years post-surgery (figure 3). The proportion of cases treated with ranibizumab increased over eightfold from $2.5 \%$ to $20.8 \%$ of all first treatments for DMO; the use of 'other treatments' showed little change, being $13 \%$ vs $10.7 \%$ (pre-surgery versus post-surgery respectively).

\section{DISCUSSION}

This real-world dataset collated from a large number of centres across the UK demonstrates the increased rate at which patients with diabetes develop DMO in the period after cataract surgery. The percentage of eyes developing first episode of 'treatment-requiring DMO' increased from $2.9 \%$ in the year immediately prior to surgery to $5.3 \%$ in the first year and $4.8 \%$ in the second year after surgery; the highest risk period was between 3 and 6 months after surgery with $1.7 \%$ of eyes developing first episode of 'treatment-requiring DMO' during that 3-month period, equating to an annualised risk of $6.8 \%$. The percentage of 'ranibizumab-requiring DMO' which in the UK is based on an OCT definition of DMO with a minimum CMT of 400 microns, increased from $0.1 \%$ for the 2 -year period prior to surgery to $1.5 \%$ of eyes for the 2-year period post-surgery.

Our findings provide additional data to suggest that there is a real increase in treatment-requiring DMO after cataract surgery. This has been a matter of considerable debate, especially since the ETDRS Report 25 failed to show a significant increase with $29 \%$ patients being noted to have CSME at their study visit occurring prior to cataract surgery and 31\% having CSME at their study visit occurring post-surgery. ${ }^{9}$ It should be noted however that this study may now be less relevant to current practice due to the modernisation of cataract surgery (including the widespread adoption of phacoemulsification since that time) and advances in the care of diabetic eye disease. Additionally the population analysed in ETDRS Report 25 differed from most studies (including ours) in that it had a very high proportion of patients with more advanced retinopathy: PDR and/or vitreous haemorrhage was present in $40 \%$ of those who had a gradeable fundus photograph from the visit immediately preceding their cataract operation. A more recent prospective study looking at unilateral cataract surgery in 132 eyes (with the 
Table 2 Cumulative risk of developing first episode of treatment-requiring diabetic macular oedema (DM0)

\begin{tabular}{|c|c|c|c|c|c|}
\hline & Time in relation to cataract surgery (months) & -24 to -12 & -24 to 0 & -24 to +12 & -24 to +24 \\
\hline \multirow{7}{*}{$\begin{array}{l}\text { Cumulative Risk of DMO } \\
(\%)\end{array}$} & All eyes & 3.1 & 6.2 & 12.1 & 14.7 \\
\hline & Stratified by ETDRS grade* & & & & \\
\hline & No DR $(n=1719)$ & 0.0 & 0.0 & 1.0 & 1.4 \\
\hline & Mild NPDR $(n=1034)$ & 2.2 & 4.8 & 10.7 & 13.4 \\
\hline & Moderate NPDR $(n=1527)$ & 5.6 & 11.8 & 23.9 & 29.5 \\
\hline & Severe NPDR $(n=165)$ & 12.6 & 26.7 & 46.0 & 59.0 \\
\hline & PDR $(n=405)$ & 6.2 & 10.9 & 16.5 & 19.1 \\
\hline
\end{tabular}

${ }^{*}$ Stratification is by ETDRS grading of retinopathy at most recent assessment prior to cataract surgery (mild, moderate and severe NPDR and PDR retinopathy). See previous table legend regarding upper boundaries of time periods.

NPDR, non-proliferative diabetic retinopathy; PDR, proliferative diabetic retinopathy.

fellow eye acting as control) noted a trend towards higher rate of CSME in the operated eye versus the fellow eye $(6.1 \%$ vs $4.5 \%)$ within the first 6 months. ${ }^{12}$ It should be noted that the difference between rates of CSME (both pre-operative and post-operative) may also reflect the inclusion of patients with milder levels of retinopathy in this later study. The findings of our study indicate that this previously observed trend towards higher levels of treatment for DMO in the post-operative phase, is indeed significant and provides, for the first time, evidence that there is a 'high-risk' period in the post-operative phase which peaks between 3 and 6 months. This dataset is taken using a single data capture platform across a large population in the UK, reflecting a wide ethnic-mix from urban and rural environments. The EMR mandates a structured assessment of DR resulting in an ETDRS grading of retinopathy and maculopathy based on the presence or absence of clinical signs at each clinic visit.

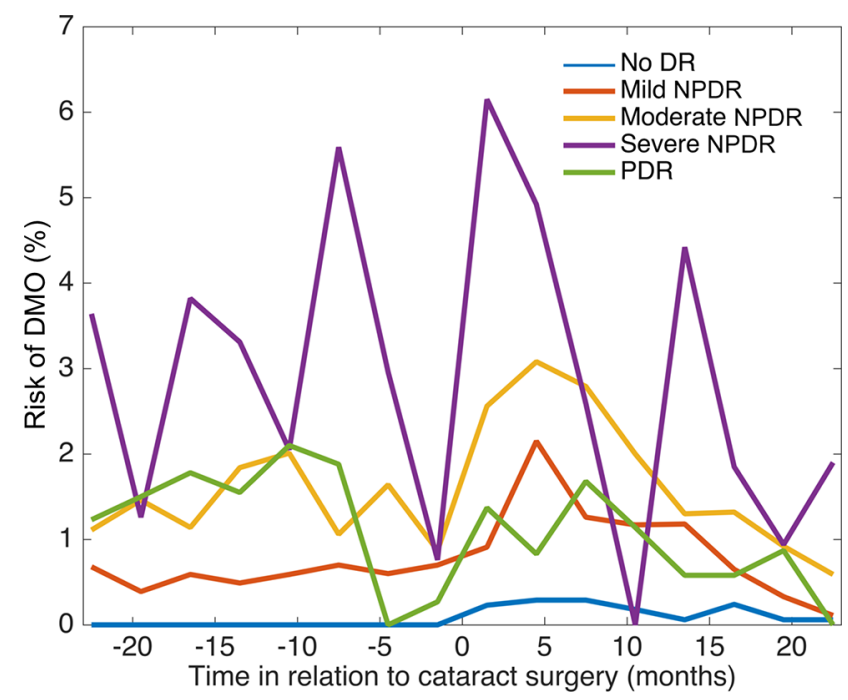

Figure 2 Effect of retinopathy on risk of developing first episode of 'Treatment-requiring diabetic macular oedema (DMO)' in relation to cataract surgery. Risk of developing first episode of 'Treatment-requiring DMO' in patients with diabetes in the 2 years before and after cataract surgery. Percentages given are of risk of 'treatment-requiring DMO' in that 3-month period. Stratification is by ETDRS grading of retinopathy at most recent assessment prior to cataract surgery (mild, moderate and severe non-proliferative diabetic retinopathy (NPDR) and proliferative diabetic retinopathy (PDR) retinopathy). The variability of the 'Severe NPDR' cohort is likely to be a function of its significantly smaller size ( $n=165)$ compared with the other cohorts $(n=1719$ for no apparent DR, $\mathrm{n}=1034$ for mild NPDR, $\mathrm{n}=1527$ for moderate NPDR, and $\mathrm{n}=405$ for proliferative DR).
It is important to note that our study is not directly comparable to earlier studies in that we are not assessing CSME, but rather 'treatment-requiring DMO'. 'Treatment-requiring DMO' is a clinically relevant group which, while subjective, provides an important metric of the burden of disease on the patient and the clinical service. The 'ranibizumab-requiring DMO' subgroup provides an objectively defined disease threshold for DMO due to the regulatory requirements in the UK requiring an OCT-defined threshold of DMO with a CMT of $>400$ microns before treatment with ranibizumab is funded. This avoids the subjectivity of clinically-defined CSME, which may show significant inter-observer variability. Both the 'treatment-requiring DMO' group and the 'ranibizumab-requiring DMO' subgroup show the same significant increase in treatment for DMO the post-operative phase, and both peak between 3 and 6 months post-operatively. It is recognised that in this analysis we have considered neither the actual level of CMT nor which instrument it has been measured on (which may influence the threshold) ${ }^{16}$; these OCT data are not currently available for our dataset but may be available in future data extractions.

The relatively modest use of ranibizumab as first line treatment in this cohort probably reflects the fact that most UK centres only adopted its use routinely after the NICE guidance in 2013.

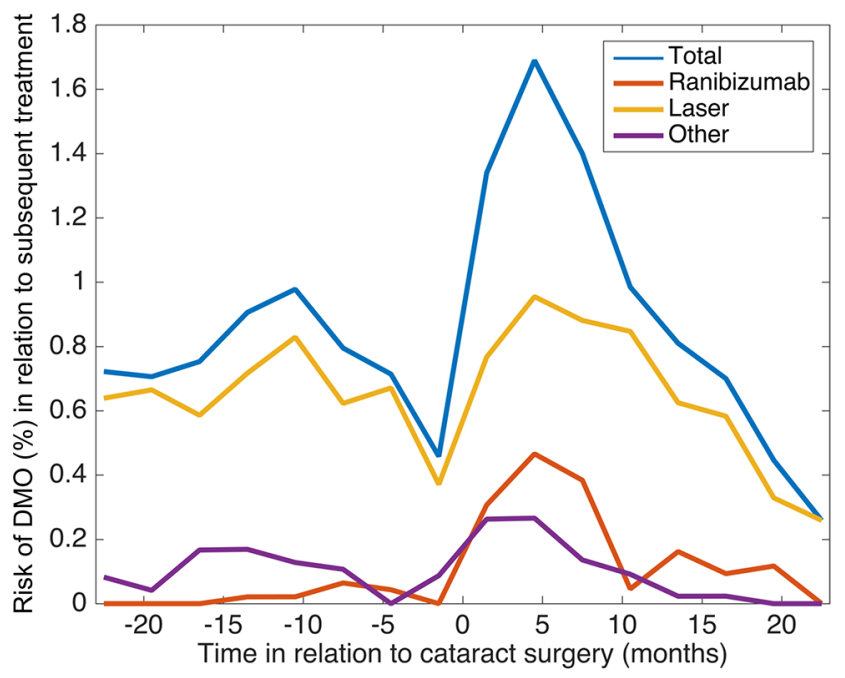

Figure 3 Treatment selection for diabetic macular oedema (DMO) over time in relation to cataract surgery. Treatment selection for first episode of 'Treatment-requiring DMO' in patients with diabetes in the 2 years before and after cataract surgery. 'Other' comprises other intravitreal treatments including alternative anti-vascular endothelial growth factor (VEGF) agents and intravitreal corticosteroid therapies. 
The increased availability of this drug at later time points of the study must be borne in mind when interpreting any increase in its usage over time, such as the fact that the proportion of cases treated with ranibizumab increased from $2.5 \%$ for the 2 years prior to surgery to $20.8 \%$ for the 2 years after surgery. In contrast any decline in usage (such as after the observed peak in the 4 to 6 month period post-surgery) can be assumed to be real (since availability would have increased over this period). The treatment data therefore reliably inform us that the risk of developing DMO exceeding 400 microns peaks in the 4 to 6 month post-operative period, which is also in line with the incidence of cases of DMO overall. Ideally a future study would take a new cohort with study inception after 2013. This would show a much higher proportion of ranibizumab (and more recently aflibercept) usage both pre-surgery and post-surgery, and would provide a more complete estimate of the proportion of 'treatment-requiring DMO' which is associated with a macular thickness of greater than 400 microns.

A possible alternative explanation when assessing studies reporting progression of diabetic retinopathy and/or maculopathy after cataract surgery is whether this simply reflects improved visualisation of the fundus. An advantage of our study is that the ranibizumab endpoint reflects an OCT-defined measure in a subset of our cohort, which is usually possible pre-surgery and post-surgery. We would however recognise two limitations. First we do not know how rigorously clinicians obeyed the $>400$ micron limit before offering ranibizumab to their patients as we have not extracted the OCT data. Second, OCT was not compulsory at all visits so there may be cases of DMO with CMT $>400$ microns which were missed and not treated. It may be argued that this was more likely to happen pre-operatively due to worse visualisation. We do not think that this was a major issue however, as most patients with worsening vision and diabetic retinopathy being considered for cataract surgery are likely to have had an OCT, although we cannot confirm the exact rates of pre-operative OCT scanning in the current data extract.

It may be difficult to distinguish pseudophakic (postoperative, cystoid macular oedema), macular oedema from DMO post-operatively; the EMR mandated structured assessment only requires recording of the location of retinal thickening. Standard clinical practice in the UK would have been to use topical steroids and/or non-steroidal drops as first line treatment if the clinician believed the problem to be pseudophakic macular oedema followed by intravitreal or sub-Tenon's triamcinolone acetonide as second line treatment. Analysis of our study suggests that this would only account for a small proportion of the cases seen. Intravitreal and sub-Tenon's triamcinolone together accounted for around one-third of the 'Other Treatment' category (specifically $12 \%$ intravitreal, $20 \%$ sub-Tenon's), and did not increase significantly after surgery with 'Other Treatments' comprising $13 \%$ vs $10.7 \%$ of total treatments (pre-surgery versus post-surgery respectively).

The lack of compulsory pre-operative and post-operative OCT and fundus fluorescein angiography (FFA) in all cases is one example of the differences between 'real-world' studies and prospective RCTs. An RCT can provide stringently quality-assured data, taken on a group of patients with the condition of interest, accompanied by minimal co-pathology and with carefully scheduled visits allowing treatment to be delivered and monitored under closely controlled conditions. Such studies have many advantages, and are valuable particularly for licensing studies as they may provide a high signal:noise ratio and, if well-designed, have a low risk of bias. They are however very expensive and generally deliberately exclude large sections of the population for whom the treatment is clinically indicated.

'Real-world' studies are of immense value in a number of situations. First, they provide some indication of how a treatment effect seen in the ideal but artificial environment of an RCT actually translate into benefits (and adverse events) in a much more heterogeneous group of patients in the less controlled environment of clinical practice. Second, they provide a mechanism of identifying and investigating rarer conditions, for which a suitably scaled RCT would be unaffordable.

Data from 'real world' studies can still be quality-assured, although not to the same level as an RCT. In our study, the design of the EMR system ensures that the data entry is structured, key fields are compulsory, and we have demonstrated previously low rates of missing non-compulsory fields. ${ }^{17}$ Fields have value cut-offs to stop major data entry errors; most minor errors may occur in either direction and so with a large sample size it is anticipated that these would become negligible. In summary, we would argue that any additional 'noise' in our study brought about by its real world, retrospective design, will be compensated for by the large sample size and multiple physicians and hospital.

One of the key questions arising from this study is whether this new evidence regarding the risk of post-operative DMO should alter practice, particularly regarding frequency (and location) of follow-up in the post-operative phase supported by the use of OCT to screen for DMO. The follow-up of patients with diabetic retinopathy after cataract surgery is very variable, being primarily dependent on the level of diabetic retinopathy. In the UK and many other countries, patients with mild NPDR may only receive the same level of hospital follow-up as patients without diabetes. Depending on local practice they receive one (or no) hospital reviews before being discharged to their community optometrist for post-operative refraction. Unless either the patient or the optometrist report specific concerns, patients may not receiveany further assessment of their fundus until their annual photographic review within the National Diabetic Eye Screening Service. It should be noted that this assessment is based on the grading of two-dimensional fundus photographs and the recorded level of visual acuity, and does not include stereo-photographs or OCT assessment which would assist in the detection of DMO. Our study suggests however that the highest risk for the development of DMO occurs in the period after the typical timing of the first post-operative visit and occurs at a significant rate even in those patients that have been assessed as having mild NPDR. It may however be possible to further stratify this risk. A DRCR. net study found that at sixteen weeks postoperatively, OCT-defined centre-involving ME developed in $0 \%$ of eyes with no preoperative DMO versus $10 \%$ if eyes had had pre-operative non-centre-involving DMO and $12 \%$ if eyes had been assessed as 'possible' centre-involving DMO. ${ }^{18}$ In this context it is also interesting to note that those patients with PDR appeared to be relatively protected from developing DMO in the post-operative phase when compared with NPDR. This is interesting but should be treated with caution as the numbers in the PDR group were significantly lower than in the other groups.

It may be argued that most patients will be aware of a decline in visual function, and so should be able to self-report visually significant DMO; indeed the NICE (UK) threshold is, in part, based on this equating to a visually significant difference. It is well known however that many patients may ignore the changes in one eye, particularly where reasonable vision is retained in the other eye. ${ }^{19}$ There may be therefore an argument for providing 
a further OCT-based assessment, whether in the community or in the hospital eye clinic, at around 4 months post-operatively to screen for the development of DMO, and to provide the opportunity for early treatment.

In summary, this analysis of a real world dataset achieved at a large number of centres across the UK indicates an increased rate of developing visually significant DMO in the post-operative phase, with a particularly high risk from 3 to 9 months. The UK requirement for an OCT-defined threshold of DMO to access ranibizumab treatment, and the presence of a clear post-operative DMO spike in this cohort provides further evidence that this is a real increase in DMO and not just an issue of visualisation. We would recommend that assessment prior to cataract surgery for any patient with diabetes should include a record of DR severity status and a macular OCT of both eyes, while recognising that the lens opacities may limit assessment in some eyes. There may also be a role for increased monitoring of patients with even relatively mild diabetic retinopathy during the post-operative phase to help detect DMO and enable earlier treatment.

\section{Author affiliations}

${ }^{1}$ University Hospitals Birmingham NHS Foundation Trust, Birmingham, UK

${ }^{2}$ The NIHR Biomedical Research Centre at Moorfields Eye Hospital NHSFT and UCL

Institute for Ophthalmology, London, UK

${ }^{3}$ Centre for Experimental Medicine, Queens University, Belfast, UK

${ }^{4}$ City University, London, UK

${ }^{5}$ Department of Ophthalmology, University of Washington, Seattle, USA

${ }^{6}$ Bristol Eye Hospital, University Hospitals Bristol NHS Foundation Trust, Bristol, UK

${ }^{7}$ Department of Ophthalmology, Hinchingbrooke Health Care NHS Trust, Huntingdon, UK

${ }^{8}$ Department of Ophthalmology, Heart of England NHS Foundation Trust, Birmingham, UK

${ }^{9}$ Department of Ophthalmology, Sheffield Teaching Hospitals NHS Foundation Trust, Sheffield, UK

${ }^{10}$ Department of Ophthalmology, Hull Royal Infirmary, Hull, UK

${ }^{11}$ Department of Ophthalmology, Peterborough and Stamford Hospitals NHS

Foundation Trust, Peterborough, UK

${ }^{12}$ Department of Ophthalmology, Calderdale and Huddersfield NHS Foundation Trust, Huddersfield, UK

${ }^{13}$ Department of Ophthalmology, Wirral University Teaching Hospital NHS Foundation Trust, Wirral, UK

${ }^{14}$ Moorfields Eye Unit, Bedford Hospitals NHS Trust, Bedford, UK

${ }^{15}$ Manchester Royal Eye Hospital, Manchester, UK

${ }^{16}$ Department of Ophthalmology, Warrington and Halton Hospitals NHS Foundation Trust, Warrington, UK

${ }^{17}$ Department of Ophthalmology, Leeds Teaching Hospitals NHS Trust, Leeds, UK

${ }^{18}$ Department of Ophthalmology, Frimley Park Hospital, Frimley, UK

${ }^{19}$ Department of Ophthalmology, Wrightington, Wigan and Leigh NHS Foundation Trust, Wigan, UK

${ }^{20}$ Department of Ophthalmology, Cambridge University Hospitals NHS Foundation Trust, Cambridge, UK

${ }^{21}$ Department of Ophthalmology, Mid Yorkshire Hospitals NHS Trust, Yorkshire, UK

${ }^{22}$ Department of Ophthalmology, Northern Devon Healthcare NHS Trust, Barnstaple, UK

${ }^{23}$ Gloucestershire Hospitals NHS Foundation Trust, Cheltenham, UK

Collaborators The full list of authors and affilliations comprising the UK DR EMR Users Group is provided in Appendix 1.

Contributors All the authors have contributed to the planning, conduct and reporting of the work described in the article.
Competing interests RLJ is the Medical Director of Medisoft Limited, the Electronic Medical Record software provider from which data were extracted.

Provenance and peer review Not commissioned; externally peer reviewed.

Data sharing statement All data have been published within this article and the accompanying manuscripts.

(C) Article author(s) (or their employer(s) unless otherwise stated in the text of the article) 2017. All rights reserved. No commercial use is permitted unless otherwise expressly granted.

\section{REFERENCES}

1 Klein BE, Klein R, Moss SE. Incidence of cataract surgery in the Wisconsin Epidemiologic Study of Diabetic Retinopathy. Am J Ophthalmol 1995;119:295-300.

2 Klein R, Klein BE, Moss SE, et al. Prevalence of diabetes mellitus in Southern Wisconsin. Am J Epidemiol 1984;119:54-61.

3 Javadi MA, Zarei-Ghanavati S. Cataracts in diabetic patients: a review article. J Ophthalmic Vis Res2008;3:52-65.

4 Patel Jl, Hykin PG, Cree IA. Diabetic cataract removal: postoperative progression of maculopathy--growth factor and clinical analysis. Br J Ophthalmol 2006;90:697-701.

5 Johnson MW. Etiology and treatment of macular edema. Am J Ophthalmol 2009;147-11-21.

6 Leal EC, Manivannan A, Hosoya K, et al. Inducible nitric oxide synthase isoform is a key mediator of leukostasis and blood-retinal barrier breakdown in diabetic retinopathy. Invest Ophthalmol Vis Sci 2007:48:5257-65.

7 Dong N, Xu B, Chu L, et al. Study of 27 aqueous humor cytokines in type 2 Diabetic patients with or without Macular Edema. PLoS One2015;10:e0125329.

8 Guex-Crosier Y. The pathogenesis and clinical presentation of macular edoema in inflammatory diseases. Doc Ophthalmol 1999;97:297-309.

9 Chew EY, Benson WE, Remaley NA, et al. Results after Lens extraction in patients with diabetic retinopathy: early treatment diabetic retinopathy study report number 25 Arch Ophthalmol 1999;117:1600-6.

10 Dowler JG, Hykin PG, Hamilton AM. Phacoemulsification versus extracapsular cataract extraction in patients with diabetes. Ophthalmology 2000;107:457-62.

11 Ostri C, Lund-Andersen H, Sander B, et al. Phacoemulsification cataract surgery in a large cohort of diabetes patients: visual acuity outcomes and prognostic factors. J Cataract Refract Surg 2011;37:2006-12.

12 Romero-Aroca P, Fernández-Ballart J, Almena-Garcia M, et al. Nonproliferative diabetic retinopathy and macular edema progression after phacoemulsification: prospective study. J Cataract Refract Surg 2006;32:1438-44.

13 Flesner P, Sander B, Henning V, et al. Cataract surgery on diabetic patients. A prospective evaluation of risk factors and complications. Acta Ophthalmol Scand 2002;80:19-24.

14 Egan C, Zhu H, Lee A, et al. The united kingdom diabetic retinopathy electronic medical record users group, report 1: baseline characteristics and visual acuity outcomes in eyes treated with intravitreal injections of ranibizumab for diabetic macular oedema. Br J Ophthalmol 2017;101:75-80.

15 NICE. Technology appraisal 274 ranibizumab for treating diabetic macular oedema (rapid review of technology appraisal guidance 237). Nice (Published Online First: 27 February 2013).

16 Kidess A, Salloukh AE, Dutt P, et al. Measurement bias between optical coherence tomography instruments can affect access to treatment: a new lottery. Clin Ophthalmol2013;7:2299-302.

17 The neovascular age-related macular degeneration database: multicenter study of 92976 ranibizumab injections: report 1: visual acuity. Ophthalmology 2014:121:1092-101.

18 Baker CW, Almukhtar T, Bressler NM, et al. Macular edema after cataract surgery in eyes without preoperative central-involved diabetic macular edema. JAMA Ophthalmol 2013:131:870-9.

19 Mitchell P, Bressler N, Tolley K, et al. Patient-reported visual function outcomes improve after ranibizumab treatment in patients with vision impairment due to diabetic macular edema: randomized clinical trial. JAMA Ophthalmo/2013;131:1339-47. 